\title{
A Small-world and Scale-free Network Generated by Sierpinski Pentagon
}

\author{
Jin Chen ${ }^{\mathrm{a}}$, Anbo Le ${ }^{\mathrm{b}}$, Qin Wang ${ }^{\mathrm{c}, *}$, Lifeng $\mathrm{Xi}^{\mathrm{b}}$ \\ ${ }^{a}$ Department of Mathematics and Statistics, Huazhong Agricultural University, 430070, Wuhan, P. R. China \\ ${ }^{b}$ Institute of Mathematics, Zhejiang Wanli University, 315100, Ningbo, P. R. China \\ ${ }^{c}$ School of Computer Science and Information Technology, Zhejiang Wanli University, 315100, Ningbo, P. R. China
}

\begin{abstract}
The Sierpinski Pentagon is used to construct evolving networks, whose nodes are all solid regular pentagons in the construction of the Sierpinski Pentagon up to the stage $t$ and any two nodes are neighbors if and only if the intersection of corresponding pentagons is non-empty and non-singleton. We show that such networks have the small-world and scale-free effects, but are not fractal scaling.
\end{abstract}

Keywords: complex network, Sierpinski Pentagon, scale-free, small-world, non-fractal scaling

\section{Introduction}

In the past two decades, lots of attention has been paid to the research of complex networks, which are ubiquitous in nature and society such as WWW [1], metabolic networks [2, 3], a network of routers connected by various physical connections [4]. Despite their diversity, most networks appearing in real world obey some organizing principles, which lead to systematic and measurable deviations basing on the random graph theory originating from Erdös and Rényi [5, 6]. In particular, two properties of real networks have aroused considerable scientific and technological interests. First, lots of measurements shows that most networks display the scale-free property [7], which means that the probability that a randomly selected node with exactly $k$ links decays as a power law, following $P(k) \sim k^{-\alpha}$, where $\alpha$ is the degree exponent. Second, lots of networks have the small-world behavior [8], namely, the geodesic distance between two uniform randomly chosen nodes grows proportionally to the logarithm of the number of nodes, simultaneously they display high average clustering coefficient.

At the mean time, various self-similar fractals are used to model and mimic evolving complex networks due to the occurrence of self-similarity in real-world complex networks demonstrated by Song et al. [9]. Zhang et al. [10, 11, 12] use the Sierpinski gasket to construct evolving networks which follow the power-law degree distribution and possess small-world effect. Several complex networks are modeled on self-similar fractals, for example, Apollonian networks [13], Koch networks [14, 15, 16, 17, 18], Platonic solids networks [19], Vicsek networks [20], Sierpinski networks [21, 22], the hierarchical networks [23] and generalized self-similar networks [24].

The fractality of complex networks is introduced in Song et al. [9, 25], see also Gallos et al. [26], In a series of articles $[27,28,29,30,31,32]$, the research group of Makse et al. analyzes the fractality of complex networks and use their techniques of fractal analysis on networks to study the biological networks. An $\ell_{B}$-box is a subset of node set $V$ such that the distance $d_{i j}$ between any pair of nodes $i$ and $j$ in the box has to be $d_{i j}<\ell_{B}$. Let $N_{B}=N_{B}\left(\ell_{B}\right)$ denote the minimal number of $\ell_{B}$-box to cover $V$, which is the same as ones in [9] and [33]. In [9], a network with node set $V$ has fractal scaling with fractal dimension $d_{B}$ if

$$
N_{B} \sim\left(\ell_{B}\right)^{-d_{B}} .
$$

In this article, we give a self-similar, scale-free and small-world complex network generated from a self-similar fractal, but our networks are non-fractal scaling, which may be a common feature for some self-similar networks as indicated by Kim et al. [33].

\footnotetext{
${ }^{*}$ Corresponding author, email: qinwang@126.com.
}

Preprint submitted to Elsevier

October 23, 2015

(C) 2015. This manuscript version is made available under the Elsevier user license

http://www.elsevier.com/open-access/userlicense/1.0/ 
In the Section 2, we give out the construction of the complex network generated by Sierpinski Pentagon. Then In the Section 3 and 4, we show that described network is scale-free and small-world. At last in Section 5, using the technique of fractal geometry, we prove that this network is not fractal scaling.

\section{Construction of the network}

\subsection{Sierpinski Pentagon and its IFS}

The Sierpinski Pentagon is generated by the following iteration function system (IFS).

$$
\begin{aligned}
& \text { Let } f_{i}(x)=\frac{3-\sqrt{5}}{2} x+b_{i}: \mathbb{R}^{2} \rightarrow \mathbb{R}^{2} \text { for } i=1,2,3,4,5 \text {, where } b_{1}=\left(\begin{array}{c}
0 \\
0
\end{array}\right), \\
& \qquad b_{2}=\left(\begin{array}{c}
\frac{\sqrt{5}-1}{2} \\
0
\end{array}\right), b_{3}=\left(\begin{array}{c}
\frac{\sqrt{5}+1}{4} \\
\frac{\sqrt{10-2 \sqrt{5}}}{4}
\end{array}\right), b_{4}=\left(\begin{array}{c}
\frac{\sqrt{5}-1}{4} \\
a
\end{array}\right), b_{5}=\left(\begin{array}{c}
\frac{\sqrt{5}-3}{4} \\
\frac{\sqrt{10-2 \sqrt{5}}}{4}
\end{array}\right),
\end{aligned}
$$

where $a=\frac{2}{3+\sqrt{5}}(2 \sin (2 \pi / 5)+\sin (\pi / 5))$, that means the ratio $\frac{3-\sqrt{5}}{2} \approx 0.382$ and

$$
b_{2} \approx\left(\begin{array}{c}
0.618 \\
0
\end{array}\right), b_{3} \approx\left(\begin{array}{c}
0.809 \\
0.588
\end{array}\right), b_{4} \approx\left(\begin{array}{c}
0.309 \\
0.951
\end{array}\right), b_{5} \approx\left(\begin{array}{c}
-0.191 \\
0.588
\end{array}\right) .
$$

Then the Sierpinski Pentagon $F$ is a self-similar fractal satisfying

$$
F=\bigcup_{i=1}^{5} f_{i}(F)
$$

Fix the solid pentagon $K$ which is the left one in Figure 1, then $f_{i}(K) \subset K$. Let $f_{6}=f_{1}$. Notice that $f_{i}(K) \cap f_{i+1}(K)$ is a singleton.
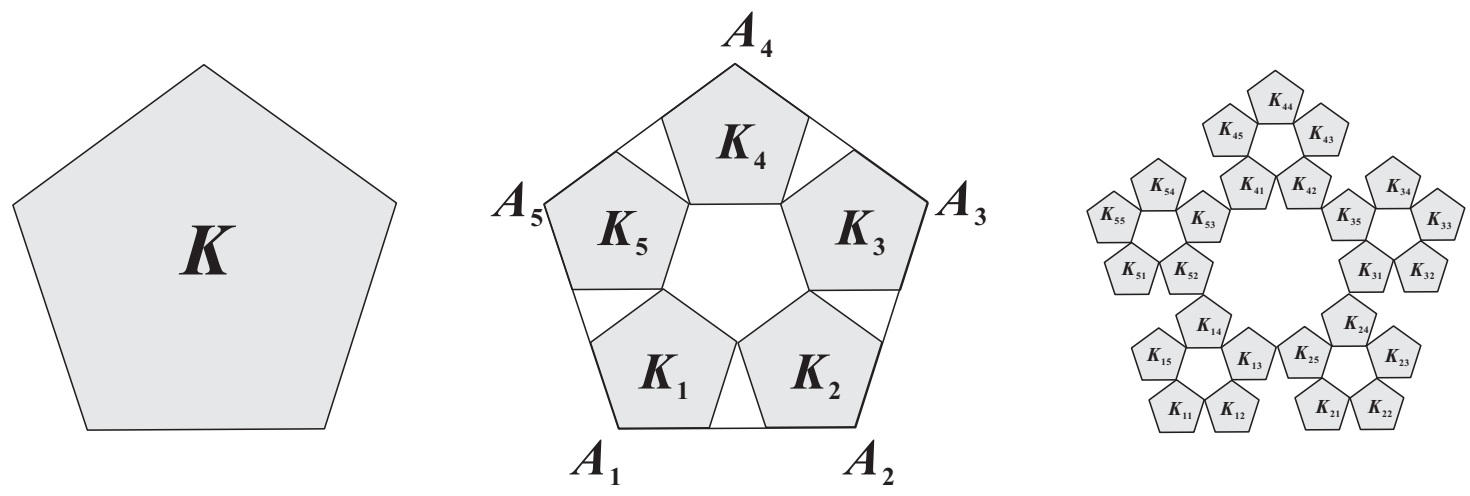

Figure 1: The first two iterations for the Sierpinski Pentagon

\subsection{Structure of Network}

Given a word $\sigma=i_{1} \cdots i_{k}$, we consider a small solid pentagon

$$
K_{\sigma}=f_{i_{1}} \circ \cdots \circ f_{i_{k}}(K) \text {. }
$$

Then its boundary $\partial K_{\sigma}$ is $f_{i_{1}} \circ \cdots \circ f_{i_{k}}(M)$, where $M=\cup_{i=1}^{5}\left[A_{i}, A_{i+1}\right]\left(A_{6}=A_{1}\right)$ and $[a, b]$ is the line segment between points $a$ and $b$ in the plane. Let $K_{\emptyset}=K$ where $\emptyset$ is the empty word.

If $\sigma=i_{1} \cdots i_{k}$ and $\tau=i_{1} \cdots i_{k} i_{k+1} \cdots i_{k+m}$ with $m \geq 1$, we denote $\sigma<\tau$. 


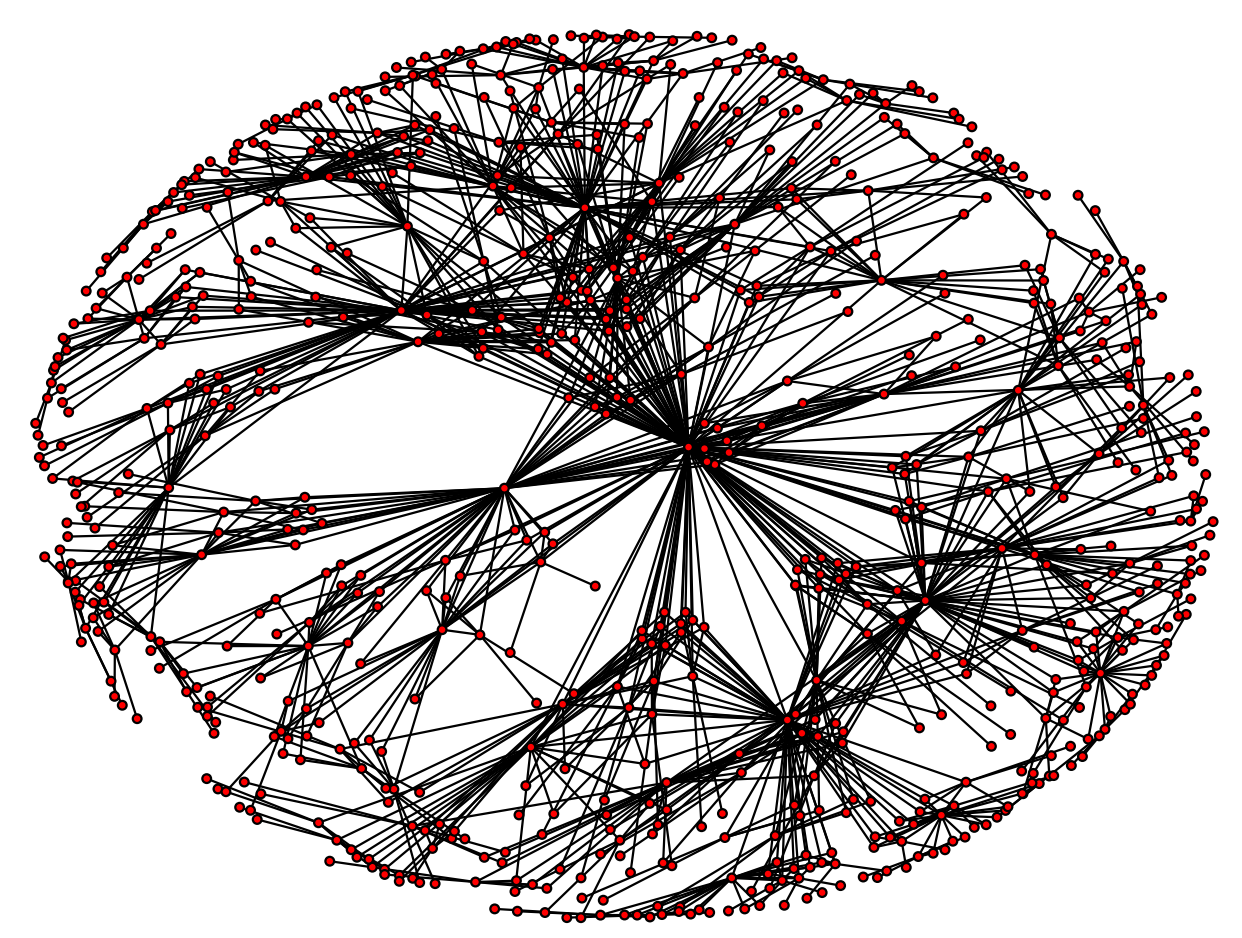

Figure 2: $G_{4}$

Fix an integer $t$, we construct a network $G_{t}$ as follows. Its nodes consist of all regular pentagons in the construction of the Sierpinski pentagons up to the stage $t$, thus its node set is identified with the set of words of length $t$

$$
V_{t}=\{\sigma: 0 \leq|\sigma| \leq t\},
$$

Since each word of length $t$ encodes such a solid regular pentagon, for two different words $\sigma$ and $\tau$, they are neighbors, denoted by $\sigma \sim \tau$, if and only if

$$
\partial K_{\sigma} \cap \partial K_{\tau} \text { is neither the empty set nor a singleton, }
$$

that means the intersection contains at least one line segment.

The total number of nodes for $G_{t}$ is

$$
\# V_{t}=1+5+5^{2}+\cdots+5^{t}=\frac{1}{4}\left(5^{t+1}-1\right) .
$$

Example 1. $1 \sim \emptyset$, since $\partial K_{1} \cap \partial K \subset\left[A_{5}, A_{1}\right] \cup\left[A_{1}, A_{2}\right]$ is not a singleton. $11,12,21,22 \sim \emptyset$, but $14,25 \nsim \emptyset$.

To illustrate the complexity of the graphs $G_{t}$, we draw $G_{4}$ in Figure 2. Now, we have the following claims.

Claim 1. If $\sigma \sim \tau$, then $\sigma<\tau$ or $\tau<\sigma$.

Notice that $\sigma \sim \emptyset$ and $\left(\partial K_{1} \cap \partial K\right) \cap\left[A_{1}, A_{2}\right] \neq \varnothing$, then $\sigma$ is consists of letters 1 and 2. Similarly, for letters $i,(i+1)$, where letter 6 is thought as 1 , we have

Claim 2. Suppose $\sigma \sim \tau$ and $\sigma<\tau$. If $\tau=\sigma j_{1} \cdots j_{k}$, then there exists $i \in\{1, \cdots, 5\}$ such that every letter appearing in $j_{1} \cdots j_{k}$ belongs to $\{i, i+1\}$.

Now, we have the following criteria of neighbors:

Proposition 1. $\sigma \sim \tau$ with $|\sigma| \leq|\tau|$ if and only if $\tau=\sigma j_{1} \cdots j_{k}$ with $k \geq 1$ and there exists $i \in\{1, \cdots, 5\}$ such that each letter $j_{p}(1 \leq p \leq k)$ belongs to $\{i, i+1\}$. 


\section{Degree formula and power-law distribution}

For $k \geq 1$, let

$$
E_{i}(k)=\left\{\sigma=j_{1} \cdots j_{k}: j_{p} \in\{i, i+1\} \text { for all } p \leq k\right\} .
$$

Then $E_{i}(k) \cap E_{j}(k) \neq \varnothing$ for $i \neq j$ if and only $|i-j|=1$, and in this case, $E_{i}(k) \cap E_{i+1}(k) \neq\left\{[i+1]^{k}\right\}$, where $[j]^{k}=\underbrace{j \cdots j}_{k}$ is a word of length $k$. Now,

$$
\#\left(\cup_{i=1}^{5} E_{i}(k)\right)=\sum_{i=1}^{5} \# E_{i}(k)-\sum_{i=1}^{5} \#\left(E_{i}(k) \cap E_{i+1}(k)\right)=5\left(2^{k}-1\right) .
$$

Let

$$
E=\bigcup_{k=1}^{\infty} \bigcup_{i=1}^{5} E_{i}(k)
$$

Let $\eta(m)=\#\{\sigma: \sigma \sim \emptyset$ and $1 \leq|\sigma| \leq m\}$. Then by Proposition 1 and (3.1), we have

$$
\eta(m)=5\left(\sum_{k=1}^{m} 2^{k}-m\right)=10 \cdot 2^{m}-5 m-10 .
$$

Given a word $\sigma=\sigma_{1} \sigma_{2} \ldots \sigma_{t} \in V_{t}$, let $T_{n}(\sigma)$ be the tail of $\sigma$ with length $n$, i.e., $T_{n}(\sigma)=\sigma_{t-n+1} \ldots \sigma_{t}$. Define

$$
q_{2}(\sigma)=\max \left\{n: T_{n}(\sigma) \subset \cup_{i=1}^{5} E_{i}(n)\right\} .
$$

Let $q_{2}(\emptyset)=0$. Therefore, $0 \leq q_{2}(\sigma) \leq t$.

Using Proposition 1, we have

Proposition 2. For any word $\sigma \in V_{t}$, we have

$$
\operatorname{deg}(\sigma)=q_{2}(\sigma)+\eta(t-|\sigma|) .
$$

Since $\eta(t-|\sigma|) \leq \operatorname{deg}(\sigma) \leq \eta(t-|\sigma|)+t$, we have

$$
10 \cdot 2^{t-|\sigma|}-5(t-|\sigma|)-10 \leq \operatorname{deg}(\sigma) \leq 10 \cdot 2^{t-|\sigma|}-5(t-|\sigma|)-10+t .
$$

Lemma 1. Suppose $t \geq 4$. We have

(1) If $0 \leq k \leq t-3$, then $\{\sigma:|\sigma| \leq k\} \subset\left\{\sigma: \operatorname{deg}(\sigma) \geq 6 \cdot 2^{t-k}\right\}$.

(2) If $t<2^{t-k}$, then $\left\{\sigma: \operatorname{deg}(\sigma) \geq 6 \cdot 2^{t-k}\right\} \subset\{\sigma:|\sigma| \leq k\}$.

(3) $\left\{\sigma: \operatorname{deg}(\sigma) \geq 6 \cdot 2^{t}\right\}=\{\emptyset\}$.

Proof. (1) When $|\sigma| \leq k \leq t-3$, since $4 \cdot 2^{x}-5 x-10 \geq 0$ for $x \geq 3$, then

$$
\begin{aligned}
\operatorname{deg}(\sigma) & \geq 10 \cdot 2^{t-|\sigma|}-5(t-|\sigma|)-10 \\
& \geq 6 \cdot 2^{t-|\sigma|} \geq 6 \cdot 2^{t-k} .
\end{aligned}
$$

(2) When $t<2^{t-k}$, suppose $|\sigma| \geq k+1$, we have

$$
\begin{aligned}
\operatorname{deg}(\sigma) & \leq 10 \cdot 2^{t-|\sigma|}-5(t-|\sigma|)-10+t \\
& \leq 10 \cdot 2^{t-k-1}+t<6 \cdot 2^{t-k} .
\end{aligned}
$$

(3) It follows from (2) for $k=0$ and $\operatorname{deg}(\emptyset) \geq 6 \cdot 2^{t}$ for any $t>3$.

Theorem 1. Suppose $t \geq 4$. If $6 \cdot 2^{t}>n>12 t$, then

$$
\left(\frac{6^{\alpha}}{10}\right) n^{-\alpha} \leq \frac{\#\left\{\sigma \in V_{t}: \operatorname{deg}(\sigma) \geq n\right\}}{\# V_{t}} \leq\left(\frac{5 \cdot 6^{\alpha}}{2}\right) n^{-\alpha} \text { with } \alpha=\frac{\log 5}{\log 2} .
$$

If $6 \cdot 2^{t} \leq n \leq \operatorname{deg}(\emptyset)$, then $\{\sigma: \operatorname{deg}(\sigma) \geq n\}=\{\emptyset\}$. 

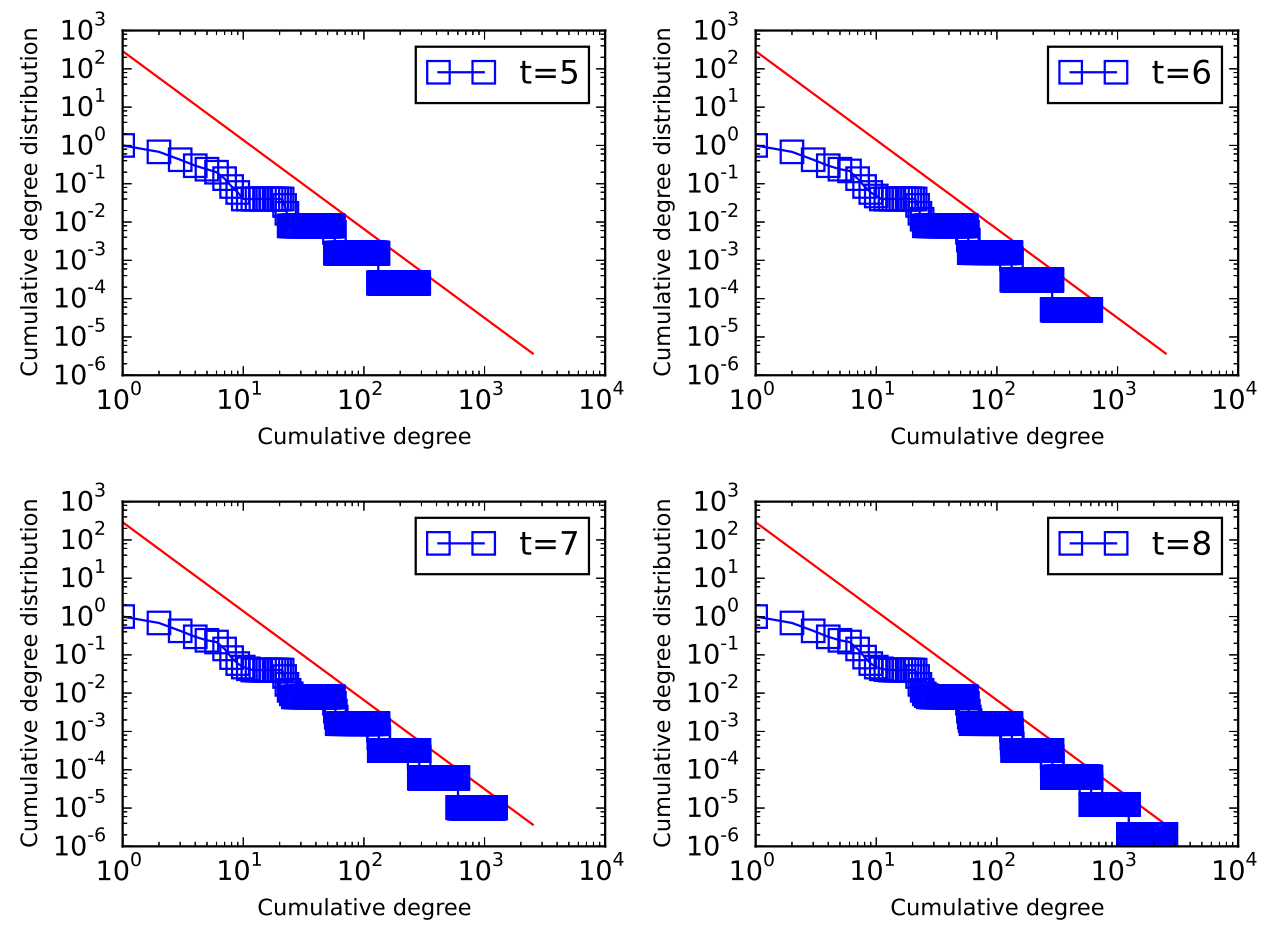

Figure 3: The $\log -\log$ graphs of the cumulative degree distributions for $t=5,6,7,8$. The slope of the solid $\operatorname{line}$ is $-\log 5 / \log 2$.

Proof. Suppose $6 \cdot 2^{t-k-1} \leq n<6 \cdot 2^{t-k}$ with $n>\max (24,12 t)$, which implies

$$
t-k \geq 3 \text { and } t<2^{t-k-1} \text {. }
$$

Now, we have

$$
\left\{\sigma: \operatorname{deg}(\sigma) \geq 6 \cdot 2^{t-k}\right\} \subset\{\sigma: \operatorname{deg}(\sigma) \geq n\} \subset\left\{\sigma: \operatorname{deg}(\sigma) \geq 6 \cdot 2^{t-k-1}\right\} .
$$

Now, $t-k \geq 3$ and $t<2^{t-k-1}$, by (1) and (2) in Lemma 1, we have

$$
\{\sigma:|\sigma| \leq k\} \subset\left\{\sigma: \operatorname{deg}(\sigma) \geq 6 \cdot 2^{t-k}\right\}
$$

and

$$
\left\{\sigma: \operatorname{deg}(\sigma) \geq 6 \cdot 2^{t-k-1}\right\} \subset\{\sigma:|\sigma| \leq k+1\}
$$

If $6 \cdot 2^{t}>n>12 t$,

$$
\frac{5^{k+1}-1}{5^{t+1}-1} \leq \frac{\#\left\{\sigma \in V_{t}: \operatorname{deg}(\sigma) \geq n\right\}}{\# V_{t}} \leq \frac{5^{k+2}-1}{5^{t+1}-1} .
$$

Here

$$
\begin{aligned}
& \frac{5^{k+1}-1}{5^{t+1}-1} \geq \frac{1}{2} \cdot \frac{5^{k+1}}{5^{t+1}}=\frac{6^{\alpha}}{10}\left(6^{-\alpha} \cdot 5^{-(t-k-1)}\right) \geq \frac{6^{\alpha}}{10}\left(6 \cdot 2^{t-k-1}\right)^{-\alpha} \geq \frac{6^{\alpha}}{10} n^{-\alpha} . \\
& \frac{5^{k+2}-1}{5^{t+1}-1} \leq \frac{1}{2} \cdot \frac{5^{k+2}}{5^{t+1}}=\frac{5 \cdot 6^{\alpha}}{2}\left(6^{-\alpha} \cdot 5^{k-t}\right) \leq \frac{5 \cdot 6^{\alpha}}{2}\left(6 \cdot 2^{t-k}\right)^{-\alpha} \leq \frac{5 \cdot 6^{\alpha}}{2} n^{-\alpha} .
\end{aligned}
$$




\section{Small-world Property}

\subsection{Average shortest path length}

For $\sigma, \tau \in V_{t}, \sigma \neq \tau$, we denote $d_{t}(\sigma, \tau)$ the geodesic distance in $G_{t}$ between $\sigma$ and $\tau$, namely the length of the shortest path in $G_{t}$ from $\sigma$ to $\tau$. Then the average shortest path length of the network $G_{t}$ is

$$
\bar{D}(t)=\frac{\sum_{\sigma \neq \tau \in V_{t}} d_{t}(\sigma, \tau)}{\# V_{t}\left(\# V_{t}-1\right) / 2},
$$

here we only compute $d_{t}(\sigma, \tau)$ or $d_{t}(\tau, \sigma)$, not both of them.

Remark 1. If $\sigma, \tau \in V_{t}$ with $\sigma=i \sigma^{\prime}$ and $\tau=i \tau^{\prime}$ for some $i \in\{1,2,3,4,5\}$, then

$$
d_{t}(\sigma, \tau)=d_{t-1}\left(\sigma^{\prime}, \tau^{\prime}\right)
$$

due to the self-similarity of $G_{t}$. Moreover, it is not hard to show that for $\sigma, \tau \in V_{t}, d_{t}(\sigma, \tau)=d_{s}(\sigma, \tau)$ for any $s \geq t$. That means we can denote $d(\sigma, \tau)$ by ignoring $t$.

Remark 2. For any $\sigma \neq \emptyset$, as in [34], let $L(\sigma)$ be the minimal number of moves for $K_{\sigma}$ to touch the boundary of $K$, i.e.,

$$
L(\sigma)=d(\sigma, \emptyset)-1
$$

For $i \neq j$, we have

$$
d\left(i \tau, j \tau^{\prime}\right) \geq L(\tau)+L\left(\tau^{\prime}\right)
$$

As in [34], we also have

$$
L(\sigma)+L(\tau) \leq L(\sigma \tau) \leq L(\sigma)+L(\tau)+1
$$

For $t \geq 1$, we denote

$$
\bar{\alpha}_{t}=\frac{\sum_{|\sigma|=t} L(\sigma)}{\#\{\sigma:|\sigma|=t\}},
$$

which is the average path moving from $K_{\sigma}$ with $|\sigma|=t$ to reach $K$. Trivially $\bar{\alpha}_{1}=0$, For $|\sigma|=2$, we can check that there are 10 pentagons $K_{\sigma}$ which are not in contact with $K$ on the boundary, which implies $L(\tau)=1$ for 10 words of length 2. Hence $\bar{\alpha}_{2}=\frac{10}{25}=\frac{2}{5}$.

Lemma 2. For $t \geq 3$, we have

$$
\bar{\alpha}_{t} \geq \frac{1}{5}(t-1) .
$$

Proof. We will prove this result by induction. Note that $\bar{\alpha}_{2}=\frac{2}{5}$. For any $k>2$, let $\sigma=\tau \sigma^{\prime}$ with $|\sigma|=k$ and $\sigma^{\prime}=k-2$, we obtain that

$$
\frac{\sum_{|\sigma|=t} L(\sigma)}{\#\{\sigma:|\sigma|=t\}}=\frac{\sum_{|\tau|=2} \sum_{\left|\sigma^{\prime}\right|=t-2} L\left(\tau \sigma^{\prime}\right)}{\sum_{|\tau|=2} \#\left\{\sigma^{\prime}: \sigma=\tau \sigma^{\prime},\left|\sigma^{\prime}\right|=t-2\right\}} .
$$

If $\tau \nsim \emptyset$, then $L(\sigma) \geq L\left(\sigma^{\prime}\right)+L(\tau)=L\left(\sigma^{\prime}\right)+1$ by (4.2), we have

$$
\frac{\sum_{\left|\sigma^{\prime}\right|=t-2} L\left(\tau \sigma^{\prime}\right)}{\#\left\{\sigma^{\prime}: \sigma=\tau \sigma^{\prime},\left|\sigma^{\prime}\right|=t-2\right\}} \geq \frac{\sum_{\left|\sigma^{\prime}\right|=t-2}\left(L\left(\sigma^{\prime}\right)+1\right)}{\#\left\{\sigma^{\prime}: \sigma=\tau \sigma^{\prime},\left|\sigma^{\prime}\right|=t-2\right\}} .
$$

If $\tau \sim \emptyset$, then $L(\sigma) \geq L\left(\sigma^{\prime}\right)+L(\tau)=L\left(\sigma^{\prime}\right)$, we have

$$
\frac{\sum_{\left|\sigma^{\prime}\right|=t-2} L\left(\tau \sigma^{\prime}\right)}{\#\left\{\sigma^{\prime}: \sigma=\tau \sigma^{\prime},\left|\sigma^{\prime}\right|=t-2\right\}} \geq \frac{\sum_{\left|\sigma^{\prime}\right|=t-2} L\left(\sigma^{\prime}\right)}{\#\left\{\sigma^{\prime}: \sigma=\tau \sigma^{\prime},\left|\sigma^{\prime}\right|=t-2\right\}} .
$$


It follows from (4.4)-(4.6) that

$$
\begin{aligned}
\bar{\alpha}_{t} & \geq \frac{\#\{|\tau|=2: L(\tau)=1\}}{25}\left(\bar{\alpha}_{t-2}+1\right)+\frac{\#|| \tau \mid=2: L(\tau)=0\}}{25} \bar{\alpha}_{t-2} \\
& =\frac{10}{25}\left(\bar{\alpha}_{t-2}+1\right)+\frac{15}{25} \bar{\alpha}_{t-2} \\
& \geq \bar{\alpha}_{t-2}+\frac{2}{5} .
\end{aligned}
$$

Thus $\bar{\alpha}_{3} \geq \bar{\alpha}_{1}+\frac{2}{5}=\frac{2}{5}$ and $\bar{\alpha}_{4} \geq \bar{\alpha}_{2}+\frac{2}{5}=\frac{4}{5}$. Therefore,

$$
\bar{\alpha}_{t} \geq \frac{1}{5}(t-1) \text { for } t=3,4 .
$$

When $t \geq 5$, by the induction hypothesis, we have $\bar{\alpha}_{t-2} \geq \frac{1}{5}(t-3)$, then

$$
\bar{\alpha}_{t} \geq \frac{1}{5}(t-3)+\frac{2}{5}=\frac{1}{5}(t-1),
$$

which ends the proof of the lemma.

Theorem 2. For any $t \geq 6$, we have

$$
\frac{128}{625}(t-2) \leq \bar{D}(t) \leq 2 t .
$$

Proof. By the criterion of neighbors, for any $\sigma \in V_{t}$, there is a path of length $\leq|\sigma|$ from the empty word $\emptyset$ to $\sigma$, i.e., $d(\sigma, \emptyset) \leq|\sigma|$. Hence, for any $\sigma, \tau \in V_{t}$,

$$
d(\sigma, \tau) \leq d(\sigma, \emptyset)+d(\emptyset, \tau) \leq|\sigma|+|\tau| \leq 2 t,
$$

which implies

$$
\bar{D}(t) \leq 2 t
$$

For $i, j \in\{1,2,3,4,5\}$ with $i<j$, by (4.2), we have

$$
\sum_{\left|\sigma^{\prime}\right|=t-1} \sum_{\left|\tau^{\prime}\right|=t-1} d_{t}\left(i \sigma^{\prime}, j \tau^{\prime}\right) \geq \sum_{\left|\sigma^{\prime}\right|=t-1} \sum_{\left|\tau^{\prime}\right|=t-1}\left(L\left(\sigma^{\prime}\right)+L\left(\tau^{\prime}\right)\right)=2 \cdot 5^{2(t-1)} \bar{\alpha}_{t-1} .
$$

Thus we have

$$
\sum_{|\sigma|=|\tau|=t, \sigma \neq \tau} d(\sigma, \tau) \geq \sum_{i, j \in\{1,2,3,4,5\}, i<j\left|\sigma^{\prime}\right|=\left|\tau^{\prime}\right|=t-1} d\left(i \sigma^{\prime}, j \tau^{\prime}\right) \geq C_{5}^{2} \cdot 2 \cdot 5^{2(t-1)} \bar{\alpha}_{t-1},
$$

which give us the lower bound for $\bar{D}(t)$, by Lemma 2 ,

$$
\bar{D}(t) \geq \frac{10 \cdot 2 \cdot 5^{2(t-1)}}{\frac{5}{2^{5}}\left(5^{t+1}-1\right)\left(5^{t}-1\right)} \bar{\alpha}_{t-1} \geq \frac{128}{125} \bar{\alpha}_{t-1} \geq \frac{128}{625}(t-2) .
$$

Then Theorem 2 follows from (4.8) and (4.9).

\subsection{Average clustering coefficient}

Given $i, j \in\{1,2,3,4,5\}$, we say that $i, j$ are dual, if $|i-j|=1$, where 1 can be thought as 6 . For example, 1 and 2,2 and 3,3 and 4,4 and 5,5 and 1 are dual respectively.

Theorem 3. If $t \geq 4$, we have

$$
\bar{C}(t)>\frac{24}{125} .
$$


Proof. For the general $G_{t}$ with $t \geq 4$, we consider the word in the form $\sigma=\sigma^{\prime} 123$, where $\left|\sigma^{\prime}\right|=t-3$. The total number of such word in $G_{t}$ is $5^{t-3}$. The criteria of neighbors implies that there are two neighbors $\sigma^{\prime} 1, \sigma^{\prime} 12$ for each $\sigma=\sigma^{\prime} 123$, and we know that $\sigma^{\prime} 1 \sim \sigma^{\prime} 12$ in $G_{t}$, thus the local clustering coefficient is 1 .

In the same way, when considering the word $\sigma^{\prime} i_{1} i_{2} i_{3}$, where $i_{2}, i_{3}$ are dual, $i_{1} \neq i_{2}$ and $i_{1} \neq i_{3}$, we notice that $\sigma^{\prime} i_{1} i_{2} i_{3}$ has two neighbors $\sigma^{\prime} i_{1}, \sigma^{\prime} i_{1} i_{2}$, and thus its local clustering coefficient is 1 .

For fixed $\sigma^{\prime}$ with $\left|\sigma^{\prime}\right|=t-3$, we have

$$
\#\left\{\sigma=\sigma^{\prime} i_{1} i_{2} i_{3}: i_{2}, i_{3} \text { are dual, } i_{1} \neq i_{2} \text { and } i_{1} \neq i_{3}\right\}=3 \cdot 2 \cdot 5=30
$$

Hence we have the following lower bound for average clustering coefficient of $G_{t}$,

$$
\bar{C}(t) \geq \frac{5^{t-3} \cdot 30}{\frac{1}{4}\left(5^{t+1}-1\right)}>\frac{24}{125}
$$

where $\# V_{t}=\frac{1}{4}\left(5^{t+1}-1\right)$.

\section{Non fractal scaling}

As in [9] and [35], an $\ell_{B}$-box is a subset of $V$ such that any two nodes have distance less than $\ell_{B}$. We let $N_{B}=$ $N_{B}\left(\ell_{B}\right)$ denote the minimal number of $\ell_{B}$-box to cover $V$, which is the same as ones in [9] and [33]. In [9], a network with node set $V$ has fractal scaling with fractal dimension $d_{B}$ if

$$
\frac{\# V}{N_{B}\left(\ell_{B}\right)} \sim\left(\ell_{B}\right)^{d_{B}},
$$

where $\frac{\# V}{N_{B}\left(\ell_{B}\right)}$ is the average number of nodes with a box of size $\ell_{B}$.

As shown in [36] by Shanker, the number of vertices in a ball or box is a very important quantity. In general, we call a network a generalized fractal network with gauge function $H$, if the average number of vertices in the box satisfies

$$
\frac{\# V}{N_{B}\left(\ell_{B}\right)} \sim H\left(\ell_{B}\right) .
$$

In particular, when $H(r)=r^{d}$, the network is a fractal network with dimension $d$ as in [9]. In Fractal Geometry [37], there are many cases where some function $H$ other than $r^{d}$ is more useful and natural, for example almost all trajectories of Brownian motion in $\mathbb{R}^{2}$ has gauge function $H(r)=r^{2} \log \log r^{-1}$, and $H(r)=r^{2} \log r^{-1} \log \log \log r^{-1}$ for $\mathbb{R}^{3}$. In [33], Kim et al. show that

$$
\frac{\# V}{N_{B}\left(\ell_{B}\right)} \sim e^{\ell_{B} / c},
$$

for many real-world networks, where $c$ is a constant.

In the computation for real-world networks, $\ell_{B}$ is usually relatively small due to the capacity of the networks and the complexity of computation, but algorithms for estimating $N_{B}$ is very sensitive to the parameter $\ell_{B}$, this makes the graphic fitting not very accurate. On the other hand, $H\left(\ell_{B}\right)$ is close to a polynomial according to the Taylor expansion when $\ell_{B}$ is relatively small. Thus the experimental results may not detect the exponential $H\left(\ell_{B}\right)$ very well. Because of this, we have to use precise mathematical analysis to estimate the minimum number $N_{B}$ of covering to obtain right conclusion for $H\left(\ell_{B}\right)$.

For our evolving network $G_{t}$ with node set $V_{t}$, we have

Theorem 4. $\frac{\# V_{t}}{N_{B}\left(\ell_{B}\right)} \sim e^{\frac{\log 5}{2} \ell_{B}}$.

Given a word $\sigma$ in $V_{t}, L(\sigma)$ is the defined as (4.1). For example, $L((23)(115))=1, L((45)(43)(2111))=$ 2, $L((4)(2)(5)(3)(1))=4$. Here

$$
L(\sigma)=d(\sigma, \emptyset)-1
$$


Notice that $d(\sigma, \emptyset)=k$, if $\sigma$ has a decomposition $\sigma=\sigma_{1} \sigma_{2} \cdots \sigma_{k}$ such that for every $i$, the word $\sigma_{i}$ is maximal suffix of $\sigma_{1} \sigma_{2} \cdots \sigma_{i}$ satisfying $\sigma_{i} \in E$. Here $E$ is defined in (3.2). Therefore,

$$
L(\sigma) \leq|\sigma|-1,
$$

and

$$
L(\sigma) \geq L\left(\sigma^{\prime}\right) \text { if } \sigma^{\prime} \text { is a suffix of } \sigma .
$$

In particular, for $\sigma^{\star}=\cdots 4253142531=i_{-\left|\sigma^{\star}\right|} \cdots i_{-1}$, where

$$
i_{-p}= \begin{cases}1 & \text { if } p \equiv 1(\bmod 5) \\ 3 & \text { if } p \equiv 2(\bmod 5) \\ 5 & \text { if } p \equiv 3(\bmod 5) \\ 2 & \text { if } p \equiv 4(\bmod 5) \\ 4 & \text { if } p \equiv 0(\bmod 5)\end{cases}
$$

In the above expression, every pair $i_{-k}$ and $i_{-(k-1)}$ are not dual, hence we have

$$
L\left(\sigma^{\star}\right)=|\sigma|-1 .
$$

As in [34], if $i \neq j$, then

$$
L(\sigma)+L(\tau) \leq d(i \sigma, j \tau) \leq L(\sigma)+L(\tau)+2
$$

and the self-similarity

$$
d(i \sigma, i \tau)=d(\sigma, \tau)
$$

Proof of Theorem 4. It suffice to show that

$$
N_{B} \sim 5^{t-\frac{\ell_{B}}{2}}
$$

(1) Firstly we give the lower bound for $N_{B}$. Let $\sigma^{\star}=\cdots 4253142531$ with the length $\left|\sigma^{\star}\right|=\left[\frac{\ell_{B}+1}{2}\right]+1$. By (5.5), $L\left(\sigma^{\star}\right)=\left[\frac{\ell_{B}+1}{2}\right]$. For any two different words $\tau, \tau^{\prime}$ of length $t-\left[\frac{\ell_{B}+1}{2}\right]$, we claim that

$$
d\left(\tau \sigma^{\star}, \tau^{\prime} \sigma^{\star}\right) \geq \ell_{B} .
$$

In fact, suppose the common prefix of $\tau, \tau^{\prime}$ is $u$, and $\tau=u i v, \tau^{\prime}=u j v^{\prime}$ with $i \neq j \in\{1,2,3,4,5\}$. It follows from (5.7), (5.6) and (5.4) that

$$
d\left(\tau \sigma^{\star}, \tau^{\prime} \sigma^{\star}\right)=d\left(i v \sigma^{\star}, j v^{\prime} \sigma^{\star}\right) \geq 2 L\left(\sigma^{\star}\right) \geq \ell_{B} .
$$

Recall that $\#\left\{\tau \sigma^{\star}:|\tau|=t-\left[\frac{\ell_{B}+1}{2}\right]-1\right\}=5^{t-\left[\frac{\ell_{B}+1}{2}\right]-1}$, using (5.8), we get the lower bound

$$
N_{B} \geq 5^{t-\left[\frac{e_{B}+1}{2}\right]-1} .
$$

(2) Now we estimate the upper bound for $N_{B}$. Given $\ell_{B} \geq 3$ and a word $\tau$ of length $\left(t-k\left[\frac{\ell_{B}-1}{2}\right]\right)$ with some integer $k \geq 1$, consider the box

$$
B_{\tau}=\left\{\tau u:|u|=\left[\frac{\ell_{B}-1}{2}\right]\right\} .
$$

In fact, by (5.7), (5.6) and (5.3), for any two different words $\tau \sigma, \tau \sigma^{\prime} \in B_{\tau}$, we have

$$
d\left(\tau \sigma, \tau \sigma^{\prime}\right)=d\left(\sigma, \sigma^{\prime}\right) \leq L(\sigma)+L\left(\sigma^{\prime}\right)+2 \leq 2(|\sigma|-1)+2=2\left[\frac{\ell_{B}-1}{2}\right]<\ell_{B},
$$

which implies

$$
\operatorname{diam}\left(B_{\tau}\right)<\ell_{B}
$$


Let $\Gamma=\left\{\tau=\emptyset\right.$ or $|\tau|=t-k\left[\frac{\ell_{B}-1}{2}\right]$ for $\left.k \in \mathbb{N}^{+}\right\}$. It is easy to know that $\left\{B_{\tau}\right\}_{\tau \in \Gamma}$ is a covering of $G_{t}$. Hence we can give the upper bound

$$
\begin{aligned}
N_{B} \leq \# \Gamma & \leq 1+5^{t-\left[\frac{\ell_{B}-1}{2}\right]}\left(1+5^{-\left[\frac{\ell_{B}-1}{2}\right]}+5^{-2\left[\frac{\ell_{B}-1}{2}\right]}+\cdots\right) \\
& \leq 1+\frac{5^{t-\left[\frac{\ell_{B}-1}{2}\right]}}{1-5^{-\left[\frac{\ell_{B}-1}{2}\right]}} \\
& \leq 1+\frac{5}{4} \cdot 5^{t-\left[\frac{\ell_{B}-1}{2}\right]}
\end{aligned}
$$

Therefore, $5^{t-\left[\frac{\ell_{B}+1}{2}\right]-1} \leq N_{B} \leq 1+\frac{5}{4} \cdot 5^{t-\left[\frac{\ell_{B}-1}{2}\right]}$, which implies $N_{B} \sim 5^{t-\frac{\ell_{B}}{2}}$.

We think the exponential gauge function $H(r)=e^{c r}$ also appears frequently in complex networks. Because the real networks have more complexity than Euclidean spaces of finite dimensions, i.e., we can not embed many real networks into Euclidean spaces, the complex network of real world is not restricted by 3-dimensional space and 1dimensional time. It may not be appropriate to describe them by the classical gauge function $r^{d}$ which is used to investigate the compact fractals in Euclidean spaces.

\section{Conclusions}

We use the Sierpinski Pentagon to construct evolving networks $G_{t},(t \geq 1)$, whose nodes is the solid regular pentagons in the construction of the Sierpinski Pentagon up to the stage $t$ and any two nodes are neighbors if and only if the intersection of corresponding pentagons is non-empty and non-singleton.

In order to show that our network is scale-free (power-law degree distribution) and have the small-world effect (small average path length and high clustering coefficient), we use the encoding method, which is commonly used in the fractal geometry, to give a criteria of neighbors and then to get the accurate degree formula for each node in $G_{t}$ by Proposition 2. Firstly, we give the degree formula to show the scale-free property of prescribed network, in fact, the power law exponent of cumulative distribution is $-\frac{\log 5}{\log 2}$ by Theorem 1 . Secondly we prove the small-world effect of $G_{t}$ by Theorems 2 and 3. At last, by Theorem 4 we show $G_{t}$ is not fractal scaling in the sense of references [9] and [25]. It should be noted that all the methods in this article are also applicable to other self-similar networks. It is interesting that the network $G_{t}$ generated from a fractal set Sierpinski Pentagon is not fractal scaling. We expect our work can provide a new view into the scale-free and small-world networks with non fractal scaling.

\section{Acknowledgement}

The work is supported by National Natural Science Foundation of China (Nos. 11371329, 11471124), NSF of Zhejiang Province (No. LR13A010001), NSF of Ningbo (No. 2010A610119), Projects in the Fundamental Research Funds for the Central Universities(No. 2662015QD016) and Projects in Science and Technique of Ningbo Municipal (No. 2012B82003).

\section{References}

[1] R. Albert, H. Jeong, and A-L Barabási, Internet: Diameter of the world-wide web, Nature 401 (1999) $130-131$.

[2] A. Wagner and D. A. Fell, The small world inside large metabolic networks, Proc. R. Soc. London B: Biol. Sci. 268 (2001)1803-1810.

[3] H.Jeong, B. Tombor, R. Albert, Z. Oltvai, and A.-L. Barábasi, The large-scale organization of metabolic networks. Nature 407 (2000): $651-654$.

[4] R. Albert, H. Jeong, H., A.-L. Barábasi, Error and attack tolerance of complex networks, nature 406 (2000) $378-382$.

[5] P. Erdős and A. Rényi, Statistical physics of social dynamics, Publ. Math. Debrecen 6 (1959) 290.

[6] B. Bollobás, Random graphs (Academic Press, London, 1985).

[7] A.-L. Barábasi and R. Albert, Emergence of scaling in random networks, science 286 (1999) 509-512..

[8] D. J. Watts and H. Strogatz, Collective dynamics of small-world networks, nature 393 (1998) 440-442.

[9] C. Song, S. Havlin, H. A. Makse, Self-similarity of complex networks, Nature 433 (2005) 392-395.

[10] Z. Zhang, S. Zhou, L. Fang, J. Guan, Y. Zhang, Maximal planar scale-free Sierpinski networks with small-world effect and power law strength-degree correlation, EPL 79 (2007) 38007. 
[11] Z. Zhang, S. Zhou, Z. Su, T. Zou, J. Guan, Random Sierpinski network with scale-free small-world and modular structure, Eur. Phys. J. B 65 (2008) 141-147.

[12] J. Guan, Y. Wu, Z. Zhang, S. Zhou, Y. Wu, A unified model for Sierpinski networks with scale-free scaling and small-world effect, Physica A 388 (12) (2009) 2571-2578.

[13] J. S. Andrade, Jr., H. J. Herrmann, R. F. S. Andrade, L. R. da Silva, Apollonian networks: Simultaneously scale-free, small world, Euclidean, space filling, and with matching graphs, Phys. Rev. Lett. 94 (2005) 018702.

[14] J.-X. Liu, X.-M. Kong, Establishment and structure properties of the scale-free Koch network, Acta Phys. Sinica 59 (2010) $2244-2249$.

[15] R. Chen, X. Fu, Q. Wu, On topological properties of the octahedral Koch network, Physica A : Statistical Mechanics and its Applications 391 (3) (2012) 880-886.

[16] M. Dai, D. Ye, J. Hou and X. Li, Scaling of average weighted receiving time on double-weighted Koch networks, Fractals (2015) 1550011.

[17] M. Dai, Q. Xie and L. Xi, Trapping on weighted tetrahedron Koch networks with small-world property, Fractals $2201 \mathrm{n} 02$ (2014) 1450006.

[18] M. Dai, D. Zhang, D. Ye, C. Zhang and L. Li, Average weighted receiving time of weighted tetrahedron Koch networks, Fractals (2015) 1550031.

[19] W.-M. Song, T. Di Matteo, T. Aste, Building complex networks with Platonic solids, Phys. Rev. E 85 (2012) 046115.

[20] Z. Zhang, S. Zhou, L. Chen, M. Yin, J. Guan, The exact solution of the mean geodesic distance for Vicsek fractals, J. Phys. A: Math. Theor. 41 (2008) 485102.

[21] L. Barriére, F. Comellas, C. Dalfó, Fractality and the small-world effect in Sierpinski graphs, J. Phys. A: Math. Gen. 39(38) (2006) 11739.

[22] A. Le, F. Gao, L. Xi, S. Yin, Complex networks modeled on the Sierpinski gasket, Physica A: Statistical Mechanics and its Applications 436 (2015) 646-657.

[23] Z. Zhang, Y. Lin, S. Gao, S. Zhou, J. Guan, Average distance in a hierarchical scale-free network: an exact solution, J. Stat. Mech. (2009) 1002.

[24] M. Knor, R. Škrekovski, Deterministic self-similar models of complex networks based on very symmetric graphs, Physica A: Statistical Mechanics and its Applications 392(19) (2013) 4629-4637.

[25] C. Song, S. Havlin, H. A. Makse, Origins of fractality in the growth of complex networks, Nat. Phys. 2 (2006) 275-281.

[26] L. K. Gallos, C. Song, H. A. Makse, A review of fractality and self-similarity in complex networks, Physica A: Statistical Mechanics and its Applications 386(2) (2007), 686-691.

[27] C. Song, L. K Gallos, S. Havlin, H. A Makse, How to calculate the fractal dimension of a complex network: the box covering algorithm, J. Stat. Mech. 2007 (2007) P03006.

[28] L. K. Gallos, C. Song, H. A. Makse, Scaling of degree correlations and its influence on diffusion in scale-free networks, Phys. Rev. Lett. 100 (2008) 248701.

[29] H. D. Rozenfeld, C. Song, H. A. Makse, Small-world to fractal transition in complex networks: A renormalization group approach, Phys. Rev. Lett. 104 (2010) 025701.

[30] V. Galvão, J. V. Miranda, R. F. S. Andrade, J. S. Andrade, Jr., L. K. Gallos, H. A. Makse, Modularity map of the network of human cell differentiation, Proc. Natl. Acad. Sci. 107(13) (2010) 5750-5755.

[31] L. K. Gallos, M. Sigman, H. A. Makse, The conundrum of functional brain networks: small-world efficiency or fractal modularity, Front Physiology 3 (2012) 00123.

[32] L. K. Gallos, H. A. Makse, M. Sigman, A small world of weak ties provides optimal global integration of self-similar modules in functional brain networks, Proc. Natl. Acad. Sci. 109(8) (2012) 2825-2830.

[33] J. S. Kim, K.-I. Goh, G. Salvi, E. Oh, B. Kahng, D. Kim, Fractality in complex networks: Critical and supercritical skeletons, Phys. Rev. E 75 (2007) 016110.

[34] F. Gao, A. Le, L. Xi, S. Yin, Asymptotic formula on average path length of fractal networks modelled on Sierpinski Gasket, arXiv:1508.00956 (2015).

[35] J.S. Kim, K.-I. Goh, B. Kahng, D. Kim, A box-covering algorithm for fractal scaling in scale-free networks, Chaos 17, (2007) 026116.

[36] O. Shanker, Defining dimension of a complex network, Mod. Phys. Lett B 21 (06) (2007), 321-326.

[37] P. Mattila, Geometry of sets and measures in Euclidean spaces: fractals and rectifiability (Cambridge University Press, 1999) 